\title{
Abordagem Anestésica na Síndrome de Kounis - Um Inimigo Cardíaco Pouco Conhecido: A Propósito de um Caso Clínico
}

\section{Anesthetic Approach in Kounis Syndrome - A Little Known Heart Enemy: Case Report}

Carolina Gonçalves ${ }^{1}$, Miguel Caridade ${ }^{1}$, Cristina Gomes ${ }^{1}$

Autor Correspondente:

Carolina Gonçalves [carolinafernandesgoncalves@gmail.com] Rua das Sete Fontes, 4710-243 Braga, Portugal

\section{RESUMO}

A síndrome de Kounis, também conhecida como síndrome da angina alérgica, foi descrita em 1991 por Kounis e Zafras como a "ocorrência coincidente de dor torácica e reação alérgica acompanhadas de achados clínicos e laboratoriais de angina pectoris clássica, causada por mediadores inflamatórios libertados durante o estímulo alérgico".

Os autores apresentam o caso de um doente de 57 anos, diagnosticado com síndrome de Kounis, submetido a polipectomia nasal, meatotomia média e etmoidectomia ântero-posterior. Com esta publicação pretende-se fazer uma revisão teórica acerca da abordagem anestésica direcionada para esta síndrome dado que se trata de um tema recente, pouco conhecido e com envolvimento multidisciplinar (Anestesiologia, Cardiologia e Medicina Intensiva).

PALAVRAS-CHAVE: Anestesia Geral/efeitos adversos; Síndrome de Kounis/etiologia

\section{ABSTRACT}

The Kounis syndrome, also known as allergic angina syndrome, was described in 1991 by Kounis and Zafras as a "coincident occurrence of chest pain and allergic reaction accompanied by clinical and laboratory findings of a classic angina pectoris caused by released inflammatory mediators during allergic stimulus".

The authors present the case of a 57-years-old male diagnosed with Kounis syndrome undergoing nasal polypectomy, meatotomy and anteroposterior ethmoidectomy. With this publication the authors pretend to make a theoretical review about the anesthetic approach directed to this syndrome since it is a recent topic with multidisciplinary involvement (Anesthesiology, Cardiology and Intensive Care).

KEYWORDS: Anesthesia, General/adverse effects; Kounis Syndrome/etiology 


\section{INTRODUÇÃO}

A síndrome de Kounis (SK), descrita em 1991 por Kounis e Zafras, é uma entidade nosológica caracterizada pela ocorrência simultânea de síndrome coronária aguda (SCA) e reação alérgica do tipo anafilática ou anafilactoide, que tem como possíveis causas fármacos, alimentos, exposições ambientais, entre outros. ${ }^{1}$

\section{CASO CLIINICO}

Doente do sexo masculino, 57 anos, proposto para cirurgia endoscópica nasossinusal com realização de polipectomia, meatotomia média e etmoidectomia ântero-posterior por pansinusite e polipose nasossinusal grau 3. Como antecedentes pessoais apresentava síndrome de Kounis, hipertensão arterial, cardiomiopatia isquémica, angina instável, dislipidemia, asma com hipersensibilidade aos anti-inflamatórios não-esteroides (tríade de Widal), síndrome ventilatória obstrutiva moderadamente grave e esofagite eosinofílica. Exame objetivo dentro dos padrões da normalidade. Não revelou alterações no estudo pré-operatório. De acordo com a classificação do estado físico da American Society of Anesthesiologists (ASA) foi classificado como ASA III.

O doente foi monitorizado de acordo com os critérios standard da ASA, BIS ${ }^{\circ}$, monitorização invasiva da pressão arterial por cateterização da artéria radial esquerda (20G) e débito urinário por algaliação vesical (16G). Foi realizada antibioterapia profilática 30 minutos antes da incisão, com 1000 mg de eritromicina intravenosa (IV). Como pré-medicação foram administrados 2 mg de midazolam, $10 \mathrm{mg}$ de metoclopramida e $50 \mathrm{mg}$ de ranitidina IV. O doente foi submetido a uma anestesia geral intravenosa. Foi iniciada a perfusão de remifentanil a 0,2 $\mathrm{mcg} / \mathrm{kg} / \mathrm{min}$ IV e procedeu-se à intubação orotraqueal com tubo orotraqueal pré-formado número 7,5 após administração IV de 200 mg de propofol e 50 mg de rocurónio permanecendo em ventilação controlada volumetricamente. A anestesia foi mantida com perfusão de propofol (100-200 mcg/kg/min) e remifentanil (100$500 \mathrm{mcg} / \mathrm{kg} / \mathrm{min})$. Iniciou, também, perfusão de dinitrato de isossorbido (1-2 mg/h). Foram, ainda, administrados $1000 \mathrm{mg}$ de paracetamol e 1,25 mg de droperidol, 30 minutos antes do final da cirurgia e $200 \mathrm{mg}$ de sugamadex ${ }^{\circledR}$. A cirurgia decorreu durante 1 hora 35 minutos, com estabilidade hemodinâmica. Perdas hemáticas quantificáveis em $100 \mathrm{~mL}$ e diurese de 0,5-1,5 mL/kg/h.

Foi extubado na sala operatória e transferido para a Unidade de Cuidados Pós-anestésicos. Ao fim de 2 horas e 10 minutos iniciou um quadro de mal-estar geral, diaforese, epistaxes, hipertensão arterial (211/116 mmHg), taquicardia (123 bpm), sem outras queixas, com retorno à estabilidade hemodinâmica após 5 mg de dinitrato de isossorbido e $5 \mathrm{mg}$ de nifedipina sublinguais. Foi posteriormente transferido para a Unidade de Cuidados Intermédios e para o internamento ao fim de 24 horas. Alta clínica no segundo dia pós-operatório.

\section{DISCUSSÃO}

A SK é caracterizada por um conjunto de sinais e sintomas que se manifestam como angina secundária a uma reação de hipersensibilidade.,., $\mathrm{A}$ sua incidência não é conhecida. ${ }^{4} \mathrm{O}$ que determina o desenvolvimento da SK continua por esclarecer; no entanto, foi sugerido que há um limiar de ativação de mastócitos e libertação de mediadores a partir do qual ocorre vasospasmo das artérias coronárias e erosão ou rutura de placas ateromatosas. Os achados clínicos sugerem que os mastócitos não só entram na lesão alvo antes da erosão da placa como também libertam o seu conteúdo antes do evento coronário agudo. ${ }^{5}$ Relatos recentes mostraram que a SK pode afetar não só as coronárias como as artérias mesentéricas e cerebrais resultando em isquemia e enfarte de órgãos vitais.. ${ }^{4}$ A libertação de renina durante episódios de anafilaxia e o seu envolvimento na disfunção cardíaca foi recentemente descrito. ${ }^{6}$

É difícil atribuir um nexo de causalidade da reação anafilática a um determinado fármaco. Nesse sentido, os testes cutâneos poderão ser úteis na identificação do agente implicado. ${ }^{?}$

De entre os fármacos diretamente envolvidos na prática anestésica que atuam como possíveis alergénios salientam-se os seguintes: etomidato, ampicilina, sulbactam, amoxicilina, amicacina, cefazolina, cefoxitina, cefuroxima, penicilina, vancomicina, ciprofloxacina, diclofenac, naproxeno, ibuprofeno, betametasona, hidrocortisona, iodopovidona, clorexidina, cisatracúrio, rocurónio, Iansoprazol, omeprazol, heparina, esmolol, insulina e enalapril, entre outros. ${ }^{1}$ Há registo de hipersensibilidade, anafilaxia e morte pela administração de sulfitos. ${ }^{8}$

Embora a SK esteja descrita maioritariamente em adultos surgiram, recentemente, novos casos na população pediátrica. ${ }^{6}$ Para o seu diagnóstico é necessário um alto índice de suspeição. Embora não seja uma doença rara, o seu diagnóstico é dificultado pela necessidade de realização de um eletrocardiograma (ECG) durante a reação alérgica. ${ }^{5}$ Dada a prioridade do tratamento da anafilaxia, quando o ECG é realizado já se encontra sem alterações. ${ }^{9}$ O seu diagnóstico baseia-se na presença de sinais e sintomas de reação alérgica em simultâneo com eventos coronários: mal-estar geral, diaforese, 
dor anginosa, hipotensão, bloqueio auriculoventricular, lipotimia, síncope, manifestações cutâneas (urticária, prurido, angioedema), respiratórias (dispneia, disfonia, estridor, sibilância) e eventualmente digestivas (dor abdominal, náuseas, vómitos). A síndrome coronária inclui angina instável com ou sem evidência de vasospasmo e/ ou enfarte agudo do miocárdio (EAM) acompanhado de alterações eletrocardiográficas, variando desde supradesnivelamento ou infradesnivelamento do segmento ST a qualquer grau de bloqueio e arritmias cardíacas, assemelhando-se a uma intoxicação digitálica. ${ }^{1,2,4,5}$ A angiografia coronária pode demonstrar espasmo das artérias coronárias, mais frequentemente da artéria coronária direita. ${ }^{2}$ A biópsia miocárdica é tipicamente normal. ${ }^{10}$ Pela diversidade do quadro, o diagnóstico é geralmente retrospetivo. No entanto, há alguns estudos que sugerem que o doseamento dos mediadores inflamatórios plasmáticos logo após o início da doença pode ajudar a evidenciar a etiologia alérgica do evento coronário, especialmente naqueles sem fatores de risco prévio ou doença coronária conhecida. Níveis elevados de histamina e triptase favorecem o diagnóstico de uma reação alérgica. ${ }^{7}$ A triptase sérica tem uma sensibilidade de 73\% e uma especificidade de 98\%, sendo o único marcador fiável. Outros marcadores inflamatórios não são úteis dado que se encontram significativamente elevados no EAM de etiologia não alérgica. 3,10

São diagnósticos diferenciais da SK o EAM, angina instável, angina de Prinzmetal e síndrome de Takotsubo. ${ }^{9}$

O tratamento da SK consiste na resolução da reação alérgica/anafilática, seguida de estabilização coronária. ${ }^{3}$ Os fármacos utilizados no tratamento específico da SCA e da anafilaxia separadamente podem constituir contraindicações quando administrados em simultâneo. ${ }^{1}$

Cevik et $a^{11}$ propõem um conjunto de recomendações para o tratamento da SK: o ácido acetilsalicílico (AAS) pode induzir anafilaxia, por isso, a sua utilidade é desconhecida; a nitroglicerina é segura; o uso de beta-bloqueadores é extremamente útil no tratamento dos SCA (no entanto, estes fármacos podem diminuir a eficácia da adrenalina); os bloqueadores dos canais de cálcio não sendo a primeira linha de tratamento nos SCA podem ser considerados o fármaco inicial em doentes com SK; os opioides devem ser usados com precaução pelo risco de desgranulação mastocitária; o uso de corticoides em doentes com evidência de reação alérgica ou na presença de sintomas refratários a altas doses de vasodilatadores é provavelmente seguro; a adrenalina, sendo o fármaco de eleição no tratamento do choque anafilático, na SK pode agravar a isquemia cardíaca. Caso se decida pela sua administração, será necessária monitorização hemodinâmica adicional e avaliação da função sistólica do ventrículo esquerdo com ecocardiograma.

No geral, a resposta foi excelente e não houve registo de mortalidade. $\bigcirc$ número de casos é limitado para alcançar conclusões definitivas acerca do tratamento, mas a maioria dos clínicos evita o uso de AAS e adrenalina. ${ }^{11}$ Admite-se que fármacos vasodilatadores são opções de primeira linha nestes doentes. ${ }^{1}$

O prognóstico depende da magnitude da reação alérgica inicial, da localização da reação antigénio-anticorpo, concentração do alergénio e a sua via de entrada e, embora seja favorável, a morte súbita não é incomum. O espasmo em múltiplos vasos pode resultar numa extensa área de miocárdio isquémico, podendo induzir acidentes cardiovasculares major, incluindo arritmias malignas e morte súbita. ${ }^{1}$

Após a revisão bibliográfica e a sua correlação com o caso clínico os autores reconhecem e reafirmam a necessidade da realização de mais estudos para conhecer a epidemiologia, diagnóstico e tratamento de uma síndrome já muito descrita e tão pouco conhecida. Entre os fármacos administrados durante o ato anestésico somente o rocurónio teria maior potencial alergénico mas, na opinião dos autores, o seu uso é justificável pelas meIhores condições de intubação. Não está claro se o episódio sucedido no período pós-operatório pode ser considerado uma apresentação de síndrome de Kounis, na medida em que não se realizou um ECG nem foi feito o doseamento de triptase durante o período das queixas, tendo sido dada prioridade ao tratamento sintomático.

\section{CONCLUSÃO}

A SK deverá ser uma hipótese diagnóstica em doentes sem fatores de risco cardiovasculares que se apresentam com um SCA, acompanhada de sintomatologia anafilática e história de exposição recente a um potencial alergénio.? Não é uma doença rara, mas é pouco diagnosticada e raramente documentada, sendo necessários mais estudos para a sua caracterização. 2,10,12

CONFLITOS DE INTERESSE: Os autores declaram não ter qualquer conflito de interesse na realização do presente trabalho.

FONTES DE FINANCIAMENTO: Não houve qualquer fonte de financiamento na realização do presente trabalho.

CONFIDENCIALIDADE DOS DADOS: Os autores declaram ter seguido os protocolos da sua instituição acerca da publicação dos dados de doentes.

PROTEÇÃO DE PESSOAS E ANIMAIS: Os autores decla- 
ram que os procedimentos seguidos na elaboração do presente trabalho estão em conformidade com as normas das comissões de investigação clínica e de ética, bem como da declaração de Helsínquia e da Associação Médica Mundial.

CONFLICTS OF INTEREST: The authors declare that they have no conflicts of interest.

FINANCIAL SUPPORT: This work has not received any contribution, grant or scholarship.

CONFIDENTIALITY OF DATA: The authors declare that they have followed the protocols of their work center on the publication of data from patients.

PROTECTION OF HUMAN AND ANIMAL SUBJECTS: The authors declare that the procedures followed were in accordance with the regulations of the relevant clinical research ethics committee and with those of the Code of Ethics of the World Medical Association (Declaration of Helsinki).

\section{REFERÊNCIAS}

1. Chang KL, Chen J, Yu J, Dou XL. Kounis syndrome leads to cardiogenic shock. Cardiovasc J Afr. 2013;24:e12-6.

2. Rico Cepeda P, Palencia Herrejón E, Rodríguez Aguirregabiria MM. Síndrome de Kounis. Med Intensiva. 2012;36:358-64.

3. Gangadharan V, Bhatheja S, Balbissi K. Kounis syndrome - an atopic monster for the heart. Cardiovasc Diagn Ther. 2013;3:47-51.

4. Davidovic G, Iric-Cupic V, Zdravkovic V, Milanov S, Dimitrijevic A. Kounis syndrome, two case reports from Kragujevac, Serbia. Am J Cardiovasc Dis. 2014;4:20-5.

5. Kounis N. Coronary hypersensitivity disorder: the Kounis syndrome. Clin Therap. 2013;35:563-71.

6. Gázquez V, Dalmau G, Gaig P, Gómez C, Navarro S, Mercé J. Kounis syndrome: report of 5 cases. J Investig Allergol Clin Immunol. 2010;20:162-5.

7. Rodrigues M, Coelho, D, Granja C. Fármacos que podem provocar síndrome de Kounis. Rev Bras Anestesiol. 2013;63:4268.

8. Kounis N, Kounis G. Anaphylactic cardiovascular collapse during anesthesia: the Kounis acute hypersensitivity syndrome seems to be the most likely cause. J Korean Med Sci. 2013;28:638-9.

9. Sanchez V, Roca L, Moreno A. Intraoperative Kounis syndrome that improved electrocardiography changes and hemodynamic situation after administering nitroglycerine. Rev Bras Anestesiol. 2014;64:281-5.

10. Lopez P, Peiris A. Kounis syndrome. South Med J. 2010;103:1148-55.

11. Cevik C, Nugent K, Shome GP, Kounis NG. Treatment of Kounis syndrome. Int J Cardiol. 2010;143:223-6.

12. Kounis N. Kounis syndrome: a monster for the atopic patient. Cardiovasc Diagn Ther. 2013;3:1-4. 